\title{
552.
}

\section{ON A DIFFERENTIAL FORMULA CONNECTED WITH THE THEORY OF CONFOCAL CONICS.}

[From the Messenger of Mathematics, vol. II. (1873), pp. 157, 158.]

THE following transformations present themselves in connexion with the theory of confocal conics.

The coordinates $x, y$ of a point are considered as functions of the parameters $h, k$ where

$$
\begin{aligned}
& \frac{x^{2}}{a+h}+\frac{y^{2}}{b+h}=1, \\
& \frac{x^{2}}{a+k}+\frac{y^{2}}{b+k}=1 ;
\end{aligned}
$$

and then assuming $\xi=x+i y, \quad \eta=x-i y(i=\sqrt{ }(-1)$ as usual), and writing $c=a-b$, we find

$$
\begin{aligned}
& h=\frac{1}{2}(-a-b+\xi \eta)+\frac{1}{2} \sqrt{ }\left\{\left(\xi^{2}-c\right)\left(\eta^{2}-c\right)\right\}, \\
& k=\frac{1}{2}(-a-b+\xi \eta)-\frac{1}{2} \sqrt{ }\left\{\left(\xi^{2}-c\right)\left(\eta^{2}-c\right)\right\},
\end{aligned}
$$

whence if

we have

$$
H=(a+h)(b+h), \quad K=(a+k)(b+k),
$$

$$
\begin{aligned}
& H=\frac{1}{4}\left\{\xi \sqrt{ }\left(\eta^{2}-c\right)+\eta \sqrt{ }\left(\xi^{2}-c\right)\right\}^{2}, \\
& K=\frac{1}{4}\left\{\xi \sqrt{ }\left(\eta^{2}-c\right)-\eta \sqrt{ }\left(\xi^{2}-c\right)\right\}^{2},
\end{aligned}
$$

or, say

$$
\begin{aligned}
& \sqrt{ }(H)=\frac{1}{2}\left\{\xi \sqrt{ }\left(\eta^{2}-c\right)+\eta \sqrt{ }\left(\xi^{2}-c\right)\right\}, \\
& \sqrt{ }(K)=\frac{1}{2}\left\{\xi \sqrt{ }\left(\eta^{2}-c\right)-\eta \sqrt{ }\left(\xi^{2}-c\right)\right\},
\end{aligned}
$$


and thence

$$
\begin{aligned}
h+\frac{1}{2}(a+b)+\sqrt{ }(H) & =\frac{1}{2}\left\{\xi+\sqrt{ }\left(\xi^{2}-c\right)\right\}\left\{\eta+\sqrt{ }\left(\eta^{2}-c\right)\right\}, \\
k+\frac{1}{2}(a+b)+\sqrt{ }(K) & =\frac{1}{2}\left\{\xi+\sqrt{ }\left(\xi^{2}-c\right)\right\}\left\{\eta-\sqrt{ }\left(\eta^{2}-c\right)\right\} \\
& =\frac{1}{2} c \frac{\xi+\sqrt{ }\left(\xi^{2}-c\right)}{\eta+\sqrt{ }\left(\eta^{2}-c\right)} .
\end{aligned}
$$

These also follow from the known differential formula

$$
4\left(d x^{2}+d y^{2}\right)=(h-k)\left(\frac{d h^{2}}{H}-\frac{d k^{2}}{K}\right)
$$

that is,

$$
\frac{4 d \xi d \eta}{\sqrt{ }\left(\xi^{2}-c\right) \sqrt{ }\left(\eta^{2}-c\right)}=\frac{d h^{2}}{H}-\frac{d k^{2}}{K}
$$

implying

$$
\begin{gathered}
\frac{2 \alpha d \xi}{\sqrt{ }\left(\xi^{2}-c\right)}=\frac{d h}{\sqrt{ }(H)}+\frac{d k}{\sqrt{ }(K)} \\
\frac{2 d \eta}{\alpha \sqrt{ }\left(\eta^{2}-c\right)}=\frac{d h}{\sqrt{ }(H)}-\frac{d k}{\sqrt{ }(K)}
\end{gathered}
$$

where $\alpha$ is a constant. The foregoing integral formulæ give at once

$$
\begin{aligned}
& \frac{d h}{\sqrt{ }(H)}=\frac{d \xi}{\sqrt{ }\left(\xi^{2}-c\right)}+\frac{d \eta}{\sqrt{ }\left(\eta^{2}-c\right)}, \\
& \frac{d k}{\sqrt{ }(K)}=\frac{d \xi}{\sqrt{ }\left(\xi^{2}-c\right)}-\frac{d \eta}{\sqrt{ }\left(\eta^{2}-c\right)},
\end{aligned}
$$

and substituting these values we find $\alpha=1$, and the differential formulæ are then satisfied.

We thence have

$$
\text { const. }=\sqrt{ }\{(a+h)(b+h)\} \pm \sqrt{ }\{(a+k)(b+k)\},
$$

as the integral of the differential equation

$$
\frac{d h}{\sqrt{ }(H)} \pm \frac{d k}{\sqrt{ }(K)}=0
$$

